\title{
A Simple Self-Adjuvanting Biomimetic Nanovaccine Self-Assembled with the Conjugate of Phospholipids and Nucleotides Can Induce Strong Cancer Immunotherapeutic Effect
}

\section{Dan Liu}

Chinese Academy of Medical Sciences \& Peking Union Medical College Institute of Biomedical Engineering

Jiale Liu

Chinese Academy of Medical Sciences \& Peking Union Medical College Institute of Biomedical Engineering

\section{Bing Ma}

Chinese Academy of Medical Sciences \& Peking Union Medical College Institute of Biomedical Engineering

\section{Bo Deng}

Chinese Academy of Medical Sciences \& Peking Union Medical College Institute of Biomedical Engineering

\section{Haiyan Xu}

Chinese Academy of Medical Sciences and Peking Union Medical College Institute of Basic Medical

\section{Sciences}

\section{Xigang Leng}

Chinese Academy of Medical Sciences \& Peking Union Medical College Institute of Biomedical Engineering

\section{Deling Kong}

Chinese Academy of Medical Sciences \& Peking Union Medical College Institute of Biomedical Engineering

\section{Lanxia Liu ( $\sim$ liulanxiabme@163.com )}

Chinese Academy of Medical Sciences \& Peking Union Medical College Institute of Biomedical Engineering https://orcid.org/0000-0002-8153-9678

\section{Research}

Keywords: self-adjuvanting, nucleotides, nanovaccine, immunotherapy 
DOI: https://doi.org/10.21203/rs.3.rs-50125/v1

License: (c) (1) This work is licensed under a Creative Commons Attribution 4.0 International License. Read Full License

Version of Record: A version of this preprint was published at Biomaterials Science on January 1st, 2021. See the published version at https://doi.org/10.1039/D0BM01333A. 


\section{Abstract}

Background: Biomimetic nanoparticles have potential applications in many fields for their favorable properties.

Results: Here, we developed a self-adjuvanting biomimetic anti-tumor nanovaccine, which was selfassembled with an amphiphilic conjugate synthetized with phospholipids of 1, 2-Dioleoyl-sn-glycero-3phosphoethanolamine (DOPE) and hydrophilic Toll-like receptor (TLR9) agonists CpG ODN. The nanovaccine could not only provide effective initial antigens stimulation and sustained long-term antigen supply with a controlled release, but also induce antigens cross-presentation via MHC-I pathway initiating $\mathrm{CD}^{+} \mathrm{T}$-cell responses. Moreover, the dense nucleotides shell around the nanovaccine could promote antigens endocytosis via various receptor-mediated pathways into dendritic cells. And CpG ODN interacted with TLR9 triggering the cytokines secretion of TNF- $a$ and IL-10 further boosted the anti-tumor humoral and cellular immune responses, which led to significant tumor suppressive effect and remarkable survival prolongation.

Conclusions: So, this nanovaccine self-assembled with phospholipid-nucleotide amphiphiles can serve as a safe, simple and efficient approach for anti-tumor immunotherapy.

\section{Background}

Nanovaccine has been investigated in recent years as an emerging field in cancer immunotherapy owing to their ability to cross biological barriers, prolong circulation times, and induce enhanced long-lasting protective immune effect [1-3]. Generally, nanovaccine carriers are required to encapsulate and codelivery antigens and adjuvants to overcome intrinsic difficulties of functional cargos and trigger delightful immune responses [4-6]. Recently, it has been reported that nucleic acids arranging in a dense spherical spatial form (SNAs) on a core made of gold or other polymers, can endow them unique properties, such as rapidly uptake through receptor-mediated mechanism and efficient protection against nuclease degradation [7-10]. However, the preparation of popular polymeric nanocarriers is complicated and the traditional core polymers have potential physiological toxicity $[6,11-14]$.

Biomimetic NPs to mimic the chemical and structural features of biological systems possess distinct properties, including favorable biocompatibility, extended circulation and targeted drug delivery. Here, we reported a simple self-adjuvanting biomimetic nanovaccine ( $\mathrm{NaVs}$ ) self-assembled with the conjugate of phospholipids and nucleotides which had a dense nucleotide shell similar to SNAs. In this study, hydrophobic biomimetic phospholipids of DOPE and hydrophilic adjuvanting Toll-like receptor (TLR9) agonists CpG ODN were exploited and covalently linked together. The resultant amphiphilic molecules similar to the phospholipid bilayer of cells membrane self-assembled forming nanocapsules. So, the nanovaccine mimicked both the chemical and structural characteristics of cells displaying good biocompatibility. Furthermore, CpG ODN not only as adjuvants enhanced the immune responses, but also the dense nucleotides shell formed with CpG ODN promoted antigens endocytosis via various receptor- 
mediated pathways and subsequently boosted the anti-tumor humoral and cellular immune responses. Therefore, the biomimetic nanovaccine offered a simple, safe and effective strategy for cancer treatment.

\section{Materials And Methods}

\subsection{Preparation of Self-Adjuvanting Biomimetic Nanovaccines}

The preparation of self-adjuvanting biomimetic nanovaccines was involved three steps. Briefly, DOPE (Sigma-Aldrich Co., USA) was pyridyldithiol-activated by 3-(2-Pyridyldithio) propionic acid Nhydroxysuccinimide ester (SPDP). Then the activated DOPE was conjugated with thiol terminated CpG ODN (Sangon Biotech Ltd., China) via a disulfide bond in the presence of phase transfer catalyst. At last, the resulted amphiphiles of DOPE-S-S-CpG ODN and ovalbumin (OVA, a model antigen, Sigma-Aldrich Co., USA) solutions were mixed and stirred at $25^{\circ} \mathrm{C}$ for the formation of self-adjuvanting biomimetic nanovaccines (named as $\mathrm{NaVs}$ ) with self-assembly. $\mathrm{NaVs}$ were washed with distilled water and lyophilized.

\subsection{Characterization of DOPE-S-S-CpG ODN Amphiphiles and $\mathrm{NaVs}$}

The conjugate of DOPE-S-S-CpG ODN was confirmed by agarose gel electrophoresis. The particle sizes and Zeta potential of NaVs were analyzed using Zetasizer Nano ZP (Malvern Instruments Ltd., UK). The redox-responsive property of NaVs was studied through observing the changes of size after reaction with excessive DL-Dithiothreitol (DTT). To investigate the stability of NaVs, the size of NaVs was measured every five days until the nanoparticles aggregated. The morphology of NaVs was observed using transmission electron microscopy (TEM) (Tecnai-F20, FEl, The Netherlands).

The OVA loading capacity of NaVs was determined using an Enhanced-BCA Protein Assay Kit. To evaluate the release behavior of antigens from NaVs in vitro, $5 \mathrm{mg}$ of lyophilized $\mathrm{NaVs}$ powder was dissolved with $1 \mathrm{~mL}$ of phosphate buffer saline (PBS, pH7.4) and placed in a dialysis bag (molecular weight: $50000 \mathrm{Da}$ ) which was immersed in $10 \mathrm{ml} \mathrm{PBS}$ at $37^{\circ} \mathrm{C}$, and the OVA concentration in dialysate were measured with BCA kits at regular intervals.

\subsection{Immunization Studies in vitro}

\subsubsection{Cytotoxicity Assessment}

Bone Marrow-Derived Dendritic Cells (BMDCs) derived from C57BL/ 6 mouse femur and cultured with IL-4 and GM-CSF (Peprotech, USA) for 6 days before further experiments. BMDCs with a density of $1 \times 10^{5}$ cells/mL were co-incubated with NaVs at a series of concentrations for $24 \mathrm{~h}$, the cytotoxicity of NaVs was evaluated using a CCK-8 kits (Dojindo Molecular Technologies Inc, Japan).

\subsubsection{Antigen Uptake in BMDCs}


To observe whether NaVs could facilitate the antigen uptake by BMDCs, after co-incubation severally with free OVA and NaVs (each concentration of FITC-OVA: $20 \mu \mathrm{g} / \mathrm{mL}$ ) for $8 \mathrm{~h}$ at $37^{\circ} \mathrm{C}$, BMDCs were washed using immunol staining wash buffer to remove the extracellular FITC-OVA or NaVs. Afterwards, BMDCs were labeled with 4, 6-diamidino-2-phenylindole (DAPI) and imaged with a laser scanning confocal microscope (Zeiss LSM 800, Germany).

\subsubsection{Endocytic Mechanism of NaVs in BMDCs}

For further exploring the endocytic pathways of DCs to NaVs, we used several uptake inhibitors to pretreat cells separately to block the corresponding endocytic pathway. Briefly, BMDCs were co-cultured with fucoidan (FCD, $10 \mu \mathrm{g} / \mathrm{mL}$ ), chlorpromazine $(C P M, 10 \mu \mathrm{g} / \mathrm{mL}$ ) or methyl- $\beta$-cyclodextrin (MBCD, $10 \mu \mathrm{g} / \mathrm{mL}$ ) for 1 hour, respectively. Afterwards, the pretreated cells were co-incubated with free FITC-OVA or NaVs (containing FITC-OVA) for hours while untreated cells were used as negative control. Then the quantity of FITC-OVA in BMDCs was detected using a flow cytometer (BD Biosciences, CA, USA) and the reduction of antigens uptake of $\mathrm{NaVs}$ compared to negative control group was calculated.

\subsubsection{Effect of NaVs on BMDCs in vitro}

In order to verify whether $\mathrm{NaVs}$ could promote BMDCs activation and antigens cross-presentation, immature BMDCs were co-cultured with PBS, free OVA, OVA + $\mathrm{CpG}$ and $\mathrm{NaVs}$ at $37^{\circ} \mathrm{C}$ in $5 \% \mathrm{CO} 2$ cell incubator for $6 \mathrm{~h}$, respectively. Then BMDCs were subsequently stained with fluorescence-labeled antibodies against CD11C, CD86, CD40 and OVA257-264 peptide SIINFEKL (Thermo Fisher Scientific Inc., USA). Events were collected and plotted using a flow cytometer. Meanwhile, the cytokines of tumor necrosis factor- $a$ (TNF-a) and interleukin-6 (IL-6) released in culture supernatants were quantified with ELISA kits (Thermo Fisher Scientific Inc., USA).

\subsection{Immunization Studies in vivo}

\subsubsection{Antigen Depot Effect}

Six-week-old female C57BL/6 mice $(n=5)$ were used to study the release behavior of NaVs in vivo. 100 $\mu \mathrm{L}$ suspension of NaVs and free OVA (each containing $20 \mu \mathrm{g}$ Cy7-labeled OVA) were intramuscularly injected into mice, respectively. Then Cy7 fluorescent signals of the immunization site were detected at predetermined intervals with the Maestro imaging system (CRI, USA) and the mean fluorescence intensity (MFI) was calculated at each checkpoint.

\subsubsection{Tumor Challenge and Therapeutic Effect}

All animal procedures were performed in accordance with the Guidelines for Care and Use of Laboratory Animals of the Chinese Academy of Medical Science and Peking Union Medical College. After getting acclimated to the SPF barrier environment, healthy female C57BL/ 6 mice were used to establish xenograft tumor models. E.G7-OVA $\left(5 \times 10^{5}\right)$ cells in logarithmic growth phase were injected subcutaneously into the right back of the mice. Until the tumor reached $5 \mathrm{~mm}$ in diameter, immunotherapy was performed on mice. Divided tumor-bearing mice into 4 groups, then respectively 
injected $50 \mu \mathrm{L}$ suspension of free OVA, OVA + CpG, NaVs (each formulation containing $20 \mu \mathrm{g}$ OVA), using PBS as a negative control. The immunization was performed three times at intervals of every other week.

To further evaluate the anti-tumor effect of NaVs, the length and width of mouse tumors were recorded with digital vernier calipers every two days, while observing the mental state of the mice. Tumor volume $\left(\mathrm{mm}^{3}\right)$ was calculated according to the following formula: (length $\times$ width $\left.{ }^{2}\right) / 2$. Two days after the last immunization, mice were sacrificed and their peripheral blood, tumors and spleens were collected immediately for the following immunological experiments. The solid tumor tissues collected from tumorbearing mice were stained with hematoxylin and eosin (H\&E) and TUNEL apoptosis kits (Beyotime Biotechnology, China) to observe the influence of NaVs on the necrosis and apoptosis of tumor cells.

\subsubsection{Secretion of OVA-Specific Antibodies}

Before mice were dislocated, the blood was drawn from the postorbital venous plexuses and blood serum were isolated through centrifugation twice at $3000 \mathrm{rpm}$ for $10 \mathrm{~min}$. Then the level of OVA-specific antibodies including IgG, IgG1, and IgG2a secreted in blood were determined by ELISA kits.

\subsubsection{T Cells Immune Responses and Immune Memory}

The spleen from the immunized mice were made into a single cell suspension and the lymphocytes were extracted. Part of the splenocytes were co-incubated with anti-mouse antibodies against CD4, CD8 and $\mathrm{CD} 3$, and detected by a flow cytometer to analyze the proliferation of T cells. The leftover cells were restimulated with OVA for 3 days and stained CD8, CD4, CD62L and CD44 antibodies labeled with different fluorescence to analyze the generation of memory $T$ cells. Meanwhile, the cell supernatant was harvested and the secretion of TNF-a and interleukin-10 (IL-10) were evaluated using ELISA kits to further confirm the type of immune responses.

\subsection{Statistical Analysis}

Data were expressed as mean \pm standard deviation (SD). The differences were assessed using ANOVA or Student's t-test and Tukey's post-test. $P$ value no more than 0.05 was considered significant.

\section{Results And Discussion}

\subsection{NaVs Preparation and Characterization}

DOPE and CpG ODN were covalently linked using SPDP as the crosslinking agent. Successful synthesis of DOPE-S-S-CpG ODN was confirmed by agarose gel electrophoresis (Figure S1). The resulted DOPE-S-SCpG ODN and OVA (as model antigen) self-assembled forming into NaVs. The morphology of NaVs was characterized by TEM and the image showed the formulations were uniform nanocapasules (Fig. 1A). The diameter of NaVs determined by dynamic light scattering (DLS) was $192.4 \pm 0.66 \mathrm{~nm}$, while the blank nanocapsule without OVA (named as SNCs) was 138.2 $\pm 0.90 \mathrm{~nm}$ (Fig. 1B and Table S1). And the size of $\mathrm{NaVs}$ remained stable in PBS solution for 45 days approximately (Fig. 1C). The loading capacity of OVA in NaVs determined by an Enhanced-BCA Protein Assay Kit was $(26.4 \pm 0.77) \%$. OVA had a burst release 
of $30 \%$ from NaVs during the first two days under conditions that mimic intracellular reducing environment (4 mM DTT in $7.4 \mathrm{pH}$ buffer) and followed with a controlled release reaching about $65 \%$ in 25 days, which was faster than the release behavior of OVA $(44.99 \pm 1.69) \%$ in the condition without DTT (Fig. 1D), indicating the NaVs did possess the redox-responsive property allowing antigens quick release after uptake into cells, and the nanovaccines could provide strong initial antigens stimuli and long-term sustained antigens exposure to immune cells which was important for eliciting powerful immune responses.

\subsection{Immunization Studies in vitro 3.2.1 Cytotoxicity Assessment}

The cell viability of BMDCs treated with NaVs at various concentrations were assessed by CCK-8 assay. According to the experimental result, even when the concentration of OVA was up to $60 \mu \mathrm{g} / \mathrm{ml}$, cell viability remained high $(102.1 \pm 5.77) \%$, which demonstrated that these nanovaccines showed excellent safety to BMDCs (Fig. 2A).

\subsubsection{Endocytosis Mechanism of NaVs}

We next investigated antigen uptake capacity of NaVs. After co-incubated with free FITC-OVA or NaVs for $8 \mathrm{~h}$ respectively, images taken with confocal fluorescence microscopy showed that much more significant OVA signal appeared in BMDCs treated with NaVs than free OVA group. Fluorescence analysis of representative image using Image $J$ software indicated that the mean fluorescence intensity (MFI) of OVA in NaVs group far exceeded that in free OVA group, which was enhanced about 6-fold. The results of the flow cytometry also demonstrated that NaVs facilitated uptake of antigens in BMDCs (93.0\% vs $17.3 \%)$ (Fig. 2B-D).

Although Chad A. Mirkin et al. reported that high density oligonucleotides on the surface of nanoparticles could bind scavenger receptors on the surface of macrophages and promote nanoparticles uptake into macrophages [10], in an attempt to further explore the endocytic pathways and investigate the mechanism of nanoparticles uptake in DCs which is important for initiating immune responses for vaccines, we pretreated BMDCs with methyl- $\beta$-cyclodextrin (MBCD), chlorpromazine (CPM) and fucoidan (FCD), respectively. MBCD can remove cholesterol from the cell membrane effectively inhibiting caveolindependent endocytosis, CPM can block the formation of clathrin-coated pits, while FCD is a pharmacological inhibitor of class A scavenger receptor (SR-A) [15-18]. As shown in Fig. 2E and 2F, compared to untreated cells, FCD led to a drastic reduction of antigens uptake of $\mathrm{NaVs}$ (dropped up to $5.2 \%$ ), MBCD reduced the uptake of cells to $36.0 \%$, while CPM caused an uptake decrease to $24.9 \%$. All the data suggested that NaVs internalized into BMDCs through multiple receptor-mediated endocytosis pathways, but mainly mediated by SR-A. Taken together, NaVs constructed with the conjugate of adjuvants CpG ODN and biomimetic materials DOPE having a "3D" structure could load antigens in the cavity of nanocapsules as vaccine delivery nanocarriers, and the high density nucleotides on the surface of nanocapsules could bind the various receptors on the surface of BMDCs and promote antigens 
endocytosis circumventing the drawbacks of free antigens, which in turn facilitated CpG ODN interaction with TLR9 receptor in endosomes and activated antigen presenting cells [19-25].

\subsubsection{BMDCs Activation and Antigens Cross-Presentation}

In order to further verify whether NaVs could promote BMDCs maturation and activation, which was essential for initiation of antigen-specific immune responses, we co-incubated immature BMDCs with PBS, Free OVA, Free OVA + CpG and NaVs for 6 h, respectively. The expression level of co-stimulatory molecules CD86 and CD40 on BMDCs treated with NaVs was nearly doubled compared to other groups (Fig. 3A and 3B). Moreover, we demonstrated that the SIINFEKL OVA-CD $8^{+}$epitope presented by MHC class I H-2Kb molecules on the surface of BMDCs treated with NaVs increased nearly 4 times compared with PBS groups (Fig. 3C), indicating that NaVs could promote antigens cross-presentation via MHC-I pathway which would subsequently initiate $\mathrm{CD} 8^{+} \mathrm{T}$ cell responses which are critical for cancer treatment, while exogenous antigens are mainly presented via the MHC-II peptide complex [26, 27]. Furthermore, the results of enzyme-linked immunosorbent assay (ELISA) of cytokines showed that BMDCs co-incubated with $\mathrm{NaVs}$ had a remarkable enhanced level of TNF-a compared with other groups (Fig. 3D) and IL-6 (Fig. 3E), especially IL-6 was enhanced up to ten-fold compared to PBS group. IL-6 can stimulate the production of chemokines and promote the activation and proliferation of $B$ cells $[28,29]$, while TNF- $a$ has important roles in stimulating $T$ cell expansion and inducing a potent tumor-specific CTLs [30, 31]. Therefore, these in vitro results showed that NaVs could promote DCs maturation, activation and antigen cross-presentation favoring induction of antigen-specific CTLs-polarizing immune responses.

\subsection{Immunization Studies in vivo}

\subsubsection{Antigen Release Behavior of NaVs in vivo}

Encouraged by the light of results in vitro, we next sought to examine antigen depot effect and release studies of NaVs in vivo using the Maestro imaging system. As shown in the Fig. 4A, fluorescence Cy7 intensity signals of free OVA reached zenith about 6 hours after injection and then decreased gradually until it is not detected at $120 \mathrm{~h}$. In contrast, the initial fluorescence intensity of NaVs increased relatively slowly and reached peak value at $12 \mathrm{~h}$ and still $25 \%$ remained at the sites after 7 days (Fig. 4B). The above results indicated that NaVs could exhibit strong initial immune stimulation and sustained longterm antigen supply in vivo.

\subsubsection{Therapeutic Effect of NaVs}

We then established E.G7-OVA tumor models and further examined the in vivo therapeutic effect of NaVs. As shown in Fig. 4C and 4E, compared with PBS group, NaVs vaccination could significantly suppress tumor growth, OVA + CpG moderately inhibited tumor growth, while free OVA only offered a slight tumor growth inhibition. In terms of survival prolongation, most the animals immunized with NaVs survived over 40 days, while mice treated with other groups almost died within 41 days (Fig. 4D). So, NaVs vaccination could offer a significant protection and remarkably prolong the life span of immunized mice. Moreover, 
the results of H\&E staining (Fig. 4F) and TUNEL apoptosis of tumor tissues (Figure S2) showed that, compared with other groups, NaVs vaccination could effectively promote necrosis and apoptosis of tumor cells.

\subsubsection{OVA-Specific Antibody Production}

In order to further verify the type of immune response triggered by the NaVs and confirm the direction of lymphocyte differentiation, we first analyze the type and amount of IgG in the blood of tumor-bearing mice which reflects the subtype of Th cells. It's generally acknowledged that the level of IgG1 antibodies are associated with Th2 immune response, whereas the production of IgG2a antibodies are characterized for Th1 responses [32,33]. As shown in Figure S3, mice immunized with the NaVs exhibited the highest OVA-specific IgG levels among all the groups, especially characterized with high IgG2a/lgG1 ratio as well, which meant NaVs polarized immune response towards Th1 type bias. In addition, Th1-dominated responses have potential for induction of CTL responses to eliminate antigen-specific tumor cells [34]. Therefore, NaVs not only had the property of significantly inducing the production of IgG antibodies, but also had the trend of eliciting Th1-type cell immune responses.

\subsubsection{T Immune Cells Activation}

For gaining a more in-depth understanding of the immune responses and relative mechanism of $\mathrm{NaVs}$ in vivo, the spleen lymphocytes of the immunized mice were stained with anti-mouse APC-CD3, FITC-CD4 and Cy5.5-CD8 antibodies. As shown in Fig. 5A, compared to other groups, the percentage of CD4 ${ }^{+} \mathrm{T}^{-}$cells from mice treated with NaVs greatly enhanced from 8.9-26.5\% (Fig. 5B), while the percentage of CD8 ${ }^{+} \mathrm{T}$ cells increased from 5.4-13.6\% (Fig. 5C and 5D). Taken together, NaVs could significantly promote the expansion of $\mathrm{CD}^{+} \mathrm{T}$ cells and $\mathrm{CD} 8^{+}$cells in the immune system of tumor-bearing mice.

Furthermore, the cytokine levels of supernatant of splenocytes after antigen re-stimulation in vitro were detected. As shown in Fig. 5E, NaVs increased the cytokine level of TNF-a approximately 4 times compared with the PBS group, which is consistent with the in vitro results. NaVs also significantly elevated the level of cytokine IL-10 (Fig. 5F). IL-10 as an anti-inflammatory factor can specifically limit Th17 inflammation to eliminate protumor environment and promote Th1-type anti-tumor immunity to recognize and eliminate tumors cells, and hence inhibit tumor development and metastasis [35, 36]. It can also activate $\mathrm{NK}$ cells and $\mathrm{CD}^{+} \mathrm{T}$ cells directly or indirectly to enhance anti-tumor effects and contribute to the formation of immune memory [37]. As stated in the previous study, here the cytokines of TNF-a and IL-10 were upregulated mainly mediated by NF-KB and AP-1 activation through mitogen-activated protein kinase (MAPK) and nuclear factor kappa-B inducing kinase -lkB kinase-lkB (NIK-IKK-IkB) pathways which resulted from TLR-9 receptor binding with the dense CpG ODNs after NaVs internalized into endosomes $[5,38]$. Altogether, we conclude that NaVs could enhance both humoral and cellular immune responses.

\subsubsection{Memory T Cell Immune Response}


An important principle of tumor nanovaccine is to stimulate the immune system producing long-lasting antigen-specific immune memory, which prevents tumor formation as well as recurrence. So, we examined the memory $T$ cell immune responses. CD44 is the most reliable marker of mouse memory cells and generally is used to define primary and memory $\mathrm{T}$ cells, while CD62L, an important adhesion molecule on central memory $T$ cells, is usually used to distinguish between central memory $T$ cells $\left(\mathrm{CD} 44^{\mathrm{Hi}} \mathrm{CD} 62 \mathrm{~L}^{\mathrm{Hi}}\right)$ and effector memory $\mathrm{T}$ cells $\left(\mathrm{CD} 44^{\mathrm{Hi}} \mathrm{CD} 62 \mathrm{~L}^{\mathrm{Lo}}\right)$ [39-41]. After the spleen lymphocytes from mice treated with $\mathrm{NaVs}$ were re-stimulated by the antigens, $\mathrm{T}_{\mathrm{CM}}$ proliferated in a large amount of both $\mathrm{CD} 4^{+} \mathrm{T}$ (PBS $6.5 \%$ vs NaVs $15.9 \%$ ) cells and CD8 ${ }^{+} \mathrm{T}$ cells (PBS $8.9 \%$ vs NaVs $18.2 \%$ ) (Fig. $5 \mathrm{G}$ and $5 \mathrm{H})$. These data suggested that NaVs could effectively induce the production and proliferation of $\mathrm{T}_{\mathrm{CM}}$, so that the body's immune system can maintain the memory of tumor antigens and provide long-term immune surveillance.

\section{Conclusions}

In summary, as shown in Fig. 6, we developed a self-adjuvanting biomimetic nanovaccine self-assembled with amphiphilic conjugate synthetized with hydrophobic phospholipids and hydrophilic TLR9 agonists CpG ODN. The nanovaccine with 3D structure could load antigens in the cavity, provide effective initial immune stimulation and sustained long-term antigen supply, and promote antigens cross-presentation via MHC-I pathway initiating CD $8^{+} \mathrm{T}$-cell responses. Moreover, the dense nucleotides shell around the nanovaccine could promote antigens endocytosis via various receptor-mediated pathways into dendritic cells. And CpG ODN interaction with TLR9 triggering the cytokines secretion of TNF- $\alpha$ and IL-10 further boosted the anti-tumor humoral and cellular immune responses as well as generated effective immune memory, which led to significant tumor suppressive effect and remarkable survival prolongation. So, the self-adjuvanting biomimetic nanovaccine can serve as a great promising immunotherapeutic approach for tumor treatment. Moreover, the method reported here could be applied to produce various types of nanovaccines for treatment of infectious diseases though loading different antigens in the cavity of the capsule, or to prepare siRNA nanocarriers for gene therapy through replacing $\mathrm{CpG}$ ODN shell around the nanovaccine with siRNA.

\section{Supplementary information}

Supplementary information accompanies this paper at Additional file 1. Additional Figures S1-S3 and Table S1

\section{Declarations}

\section{Abbreviations}

DOPE: 1, 2-Dioleoyl-sn-glycero-3-phosphoethanolamine; TLR9: Toll-like receptor; SNAs: spherical nucleic acids; NaVs: self-adjuvanting biomimetic nanovaccines; SPDP: 3-(2-Pyridyldithio) propionic acid N- 
hydroxysuccinimide ester; OVA: ovalbumin; DTT: DL-Dithiothreitol; TEM: transmission electron microscopy; BMDCs: bone marrow-derived dendritic Cells; DAPI: 4, 6-diamidino-2-phenylindole; LSM: laser scanning confocal microscope; FCD: fucoidan; CPM: chlorpromazine; MBCD: methyl- $\beta$-cyclodextrin; TNFa: tumor necrosis factor-a; IL-6: interleukin-6; MFI: mean fluorescence intensity; H\&E: hematoxylin and eosin; IL-10: interleukin-10; SD: standard deviation; DLS: dynamic light scattering; SR-A: class A scavenger receptor; SNC: Spherical nano capsule; MAPK: mitogen-activated protein kinase; NIK-IKK-IkB: nuclear factor kappa-B inducing kinase -lkB kinase-lkB.

\section{Declarations}

\section{Acknowledgements}

Not applicable.

\section{Authors' contributions}

LL designed the study. DL and JL performed the experiments and analysis data.

$B M$ and BD participated in the animal experiments. LL revised figures and prepared the manuscript. HX, $\mathrm{XL}$ and $\mathrm{DK}$ reviewed and revised the manuscript. All authors have read and approved the final manuscript.

\section{Funding}

This work was supported by the Natural Science Foundation of China (No. 31870920), CAMS Innovation Fund for Health and longevity pilot project (Youth Award Program, 2019-RC-HL-015) and CAMS Innovation Fund for Medical Sciences (CAMS-12M-3-004).

\section{Availability of data and materials}

All data generated or analyzed during this study are included in this published article.

\section{Ethics approval and consent to participate}

All animal procedures were performed in accordance with the Guidelines for Care and Use of Laboratory Animals of the Chinese Academy of Medical Science and Peking Union Medical College and were approved by the Animal Ethics Committee of the Chinese Academy of Medical Science and Peking Union Medical College.

\section{Consent for publication}

All authors have agreed to publish this article.

\section{Competing interests}


The authors declare no conflict of interest.

\section{Author details}

1. The Tianjin Key Laboratory of Biomaterials, Institute of Biomedical Engineering, Peking Union Medical College \& Chinese Academy of Medical Sciences, Tianjin 300192, China

2. Institute of Basic Medical Sciences, Chinese Academy of Medical Sciences, School of Basic Medicine, Peking Union Medical College, Beijing100730, China

3. College of Life Science, Nankai University, Tianjin 300071, China.

\section{References}

1. Acharya S, Sahoo SK. PLGA nanoparticles containing various anticancer agents and tumour delivery by EPR effect. Adv Drug Del Rev. 2011;63:170-83.

2. Shao K, Singha S, Clemente-Casares X, Tsai S, Yang Y, Santamaria P. Nanoparticle-based immunotherapy for cancer. ACS Nano. 2015;9:16-30.

3. Zhou J, Kroll AV, Holay M, Fang RH, Zhang L. Biomimetic Nanotechnology toward Personalized Vaccines. Advanced materials (Deerfield Beach, Fla) 2019:e1901255.

4. Zhu G, Zhang F, Ni Q, Niu G, Chen X. Efficient Nanovaccine Delivery in Cancer Immunotherapy. ACS Nano. 2017;11:2387-92.

5. Luo M, Wang H, Wang Z, Cai H, Lu Z, Li Y, Du M, Huang G, Wang C, Chen X, et al. A STING-activating nanovaccine for cancer immunotherapy. Nature nanotechnology. 2017;12:648-54.

6. Singha S, Shao K, Ellestad KK, Yang Y, Santamaria P. Nanoparticles for Immune Stimulation Against Infection, Cancer, and Autoimmunity. ACS Nano. 2018;12:10621-35.

7. Mirkin CA, Letsinger RL, Mucic RC, Storhoff JJ. A DNA-based method for rationally assembling nanoparticles into macroscopic materials. Nature. 1996;382:607-9.

8. Mirkin CA, Petrosko SH. Spherical Nucleic Acids: Adding a New Dimension to Nucleic Acids and Clinical Chemistry. Clin Chem. 2018;64:971-2.

9. Ferrer JR, Sinegra AJ, Ivancic D, Yeap XY, Qiu L, Wang J-J, Zhang ZJ, Wertheim JA, Mirkin CA. Structure-Dependent Biodistribution of Liposomal Spherical Nucleic Acids. ACS nano 2020:10.1021/acsnano.1029b07254.

10. Choi CHJ, Hao L, Narayan SP, Auyeung E, Mirkin CA. Mechanism for the endocytosis of spherical nucleic acid nanoparticle conjugates. Proc Natl Acad Sci U S A. 2013;110:7625-30.

11. Wang Y, Miao L, Satterlee A, Huang L. Delivery of oligonucleotides with lipid nanoparticles. Adv Drug Del Rev. 2015;87:68-80.

12. Giodini L, Re FL, Campagnol D, Marangon E, Posocco B, Dreussi E, Toffoli G. Nanocarriers in cancer clinical practice: a pharmacokinetic issue. Nanomedicine. 2017;13:583-99. 
13. Xin Y, Huang M, Guo WW, Huang Q, Zhang LZ, Jiang G. Nano-based delivery of RNAi in cancer therapy. Mol Cancer. 2017;16:134.

14. Shi D, Bedford NM, Cho H-S. Engineered multifunctional nanocarriers for cancer diagnosis and therapeutics. Small. 2011;7:2549-67.

15. De Koker S, De Geest BG, Singh SK, De Rycke R, Naessens T, Van Kooyk Y, Demeester J, De Smedt SC, Grooten J. Polyelectrolyte microcapsules as antigen delivery vehicles to dendritic cells: uptake, processing, and cross-presentation of encapsulated antigens. Angew Chem. 2009;48:8485-9.

16. Patel PC, Giljohann DA, Daniel WL, Zheng D, Prigodich AE, Mirkin CA. Scavenger receptors mediate cellular uptake of polyvalent oligonucleotide-functionalized gold nanoparticles. Bioconjugate Chem. 2010;21:2250-6.

17. Rejman J, Bragonzi A, Conese M. Role of clathrin- and caveolae-mediated endocytosis in gene transfer mediated by lipo- and polyplexes. Mol Ther. 2005;12:468-74.

18. Kuhn DA, Vanhecke D, Michen B, Blank F, Gehr P, Petri-Fink A, Rothen-Rutishauser B. Different endocytotic uptake mechanisms for nanoparticles in epithelial cells and macrophages. Beilstein journal of nanotechnology. 2014;5:1625-36.

19. Hanagata N. CpG oligodeoxynucleotide nanomedicines for the prophylaxis or treatment of cancers, infectious diseases, and allergies. Int J Nanomed. 2017;12:515-31.

20. Murad YM, Clay TM. CpG oligodeoxynucleotides as TLR9 agonists: therapeutic applications in cancer. Biodrugs. 2009;23:361-75.

21. Hemmi H, Takeuchi O, Kawai T, Kaisho T, Sato S, Sanjo H, Matsumoto M, Hoshino K, Wagner H, Takeda K, Akira S. A Toll-like receptor recognizes bacterial DNA. Nature. 2000;408:740-5.

22. Kuai R, Sun X, Yuan W, Ochyl LJ, Xu Y, Hassani Najafabadi A, Scheetz L, Yu M-Z, Balwani I, Schwendeman A, Moon JJ. Dual TLR agonist nanodiscs as a strong adjuvant system for vaccines and immunotherapy. J Controlled Release. 2018;282:131-9.

23. Barber GN. Cytoplasmic DNA innate immune pathways. Immunol Rev 2011, 243.

24. Takeshita F, Gursel I, Ishii KJ, Suzuki K, Gursel M, Klinman DM. Signal transduction pathways mediated by the interaction of CpG DNA with Toll-like receptor 9. Semin Immunol. 2004;16:17-22.

25. Yue J, Pallares RM, Cole LE, Coughlin EE, Mirkin CA, Lee A, Odom TW. Smaller CpG-Conjugated Gold Nanoconstructs Achieve Higher Targeting Specificity of Immune Activation. ACS Appl Mater Interfaces. 2018;10:21920-6.

26. Joffre OP, Segura E, Savina A, Amigorena S. Cross-presentation by dendritic cells. Nature reviews Immunology. 2012;12:557-69.

27. Gardner A, Ruffell B. Dendritic Cells and Cancer Immunity. Trends Immunol. 2016;37:855-65.

28. Grivennikov SI, Karin M. Inflammatory cytokines in cancer: tumour necrosis factor and interleukin 6 take the stage. Ann Rheum Dis. 2011;70(Suppl 1):i104-8.

29. Jones SA, Jenkins BJ. Recent insights into targeting the IL-6 cytokine family in inflammatory diseases and cancer. Nature reviews Immunology. 2018;18:773-89. 
30. Balkwill F. TNF-alpha in promotion and progression of cancer. Cancer Metastasis Rev. 2006;25:40916.

31. Balkwill F. Tumour necrosis factor and cancer. Nature reviews Cancer. 2009;9:361-71.

32. Liu J, Liu X, Han Y, Zhang J, Liu D, Ma G, Li C, Liu L, Kong D. Nanovaccine Incorporated with Hydroxychloroquine Enhances Antigen Cross-Presentation and Promotes Antitumor Immune Responses. ACS Appl Mater Interfaces. 2018;10:30983-93.

33. Heeg K, Zimmermann S. CpG DNA as a Th1 trigger. Int Arch Allergy Immunol. 2000;121:87-97.

34. Romagnani S. T-cell subsets (Th1 versus Th2). Annals of allergy, asthma \& immunology: official publication of the American College of Allergy, Asthma, \& Immunology 2000, 85.

35. Saraiva M, O'Garra A. The regulation of IL-10 production by immune cells. Nature reviews Immunology. 2010;10:170-81.

36. Oft M. IL-10: master switch from tumor-promoting inflammation to antitumor immunity. Cancer immunology research. 2014;2:194-9.

37. Naing A, Infante JR, Papadopoulos KP, Chan IH, Shen C, Ratti NP, Rojo B, Autio KA, Wong DJ, Patel MR, et al: PEGylated IL-10 (Pegilodecakin) Induces Systemic Immune Activation, CD8 T Cell Invigoration and Polyclonal T Cell Expansion in Cancer Patients. Cancer Cel/ 2018, 34.

38. Wu H-M, Wang J, Zhang B, Fang L, Xu K, Liu R-Y. CpG-ODN promotes phagocytosis and autophagy through JNK/P38 signal pathway in Staphylococcus aureus-stimulated macrophage. Life Sci. 2016;161:51-9.

39. Kaech SM, Cui W. Transcriptional control of effector and memory CD8 + T cell differentiation. Nature reviews Immunology. 2012;12:749-61.

40. Sallusto F, Geginat J, Lanzavecchia A. Central memory and effector memory T cell subsets: function, generation, and maintenance. Annu Rev Immunol. 2004;22:745-63.

41. Liu L, Ma P, Wang H, Zhang C, Sun H, Wang C, Song C, Leng X, Kong D, Ma G. Immune responses to vaccines delivered by encapsulation into and/or adsorption onto cationic lipid-PLGA hybrid nanoparticles. J Controlled Release. 2016;225:230-9.

\section{Figures}


A

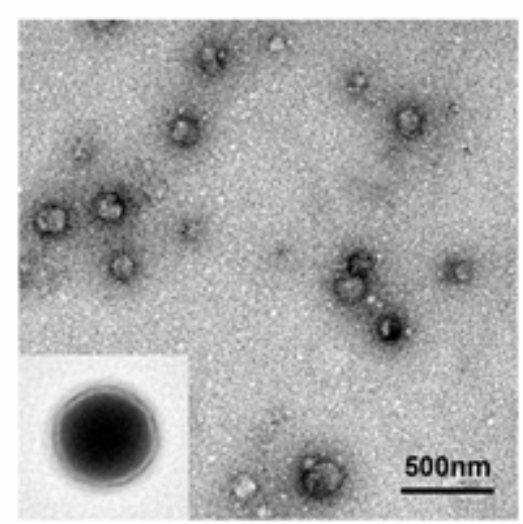

C

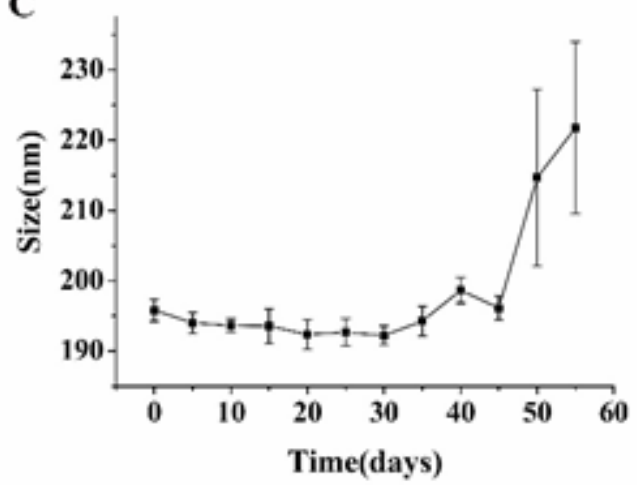

B

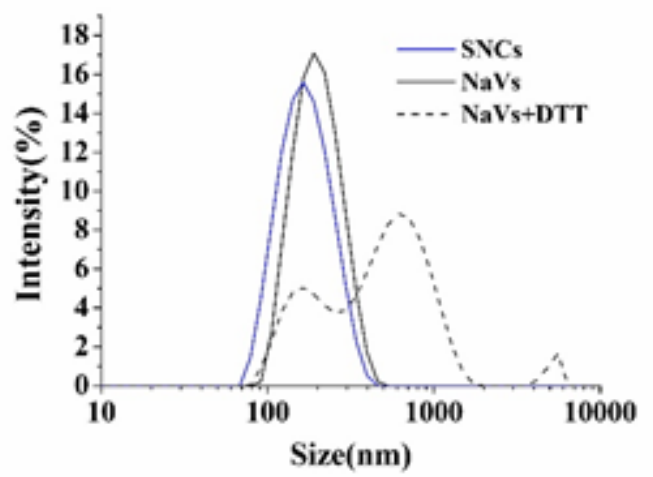

D

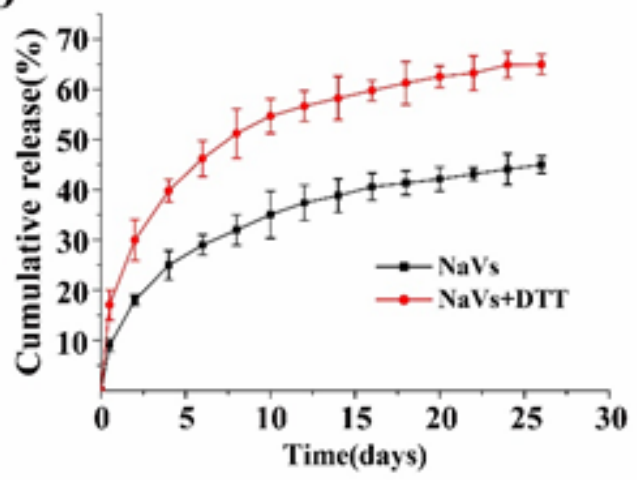

Figure 1

(A) Typical TEM images of NaVs. (B) The particle distribution of different formations, (C) and stability study of NaVs in vitro, and (D) In vitro OVA release profile from NPs with/without DTT reaction in $7.4 \mathrm{pH}$ buffer. 
A

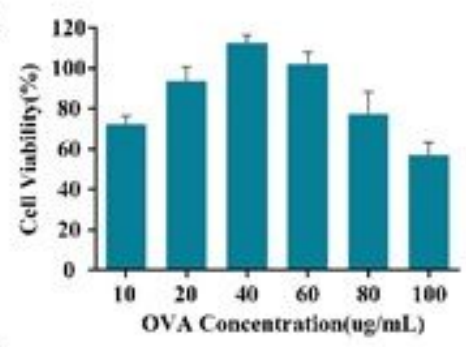

C

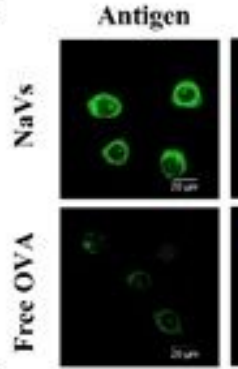

E

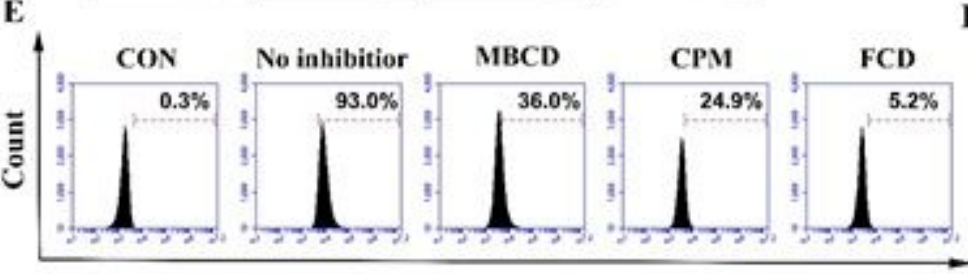

B

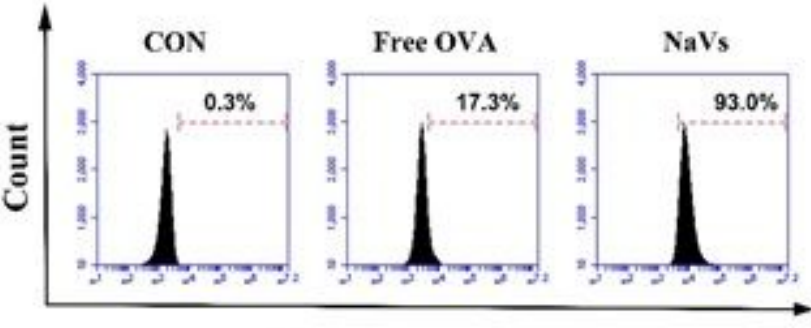

Brightfield D
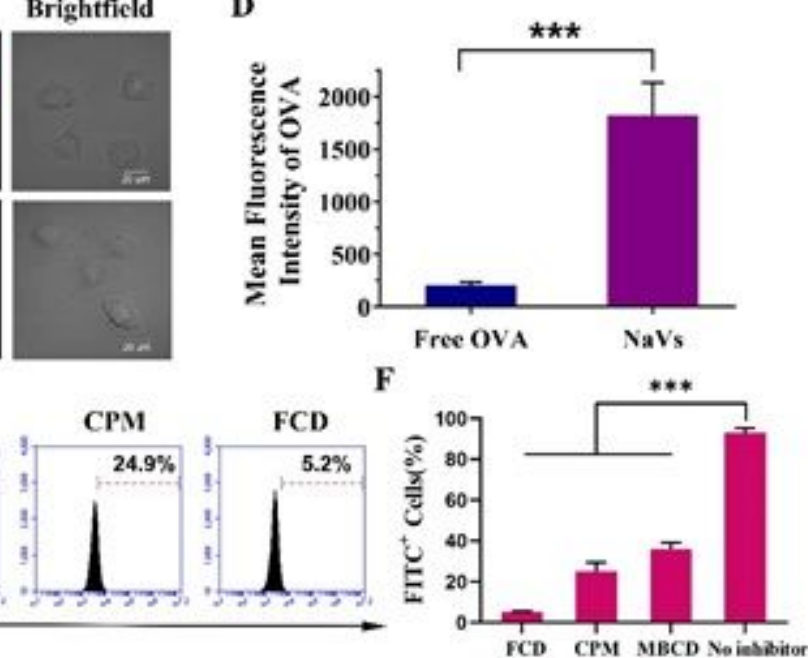

Figure 2

Studies on cytotoxicity and cellular uptake pathways of nanoparticles. (A) Cell viability at different concentrations of OVA. (B) Representative flow cytometry analysis of cellular uptake of NaVs and free OVA. (C) CLSM images of BMDCs after incubation with free FITC-OVA and NaVs and (D) representative image of mean fluorescence intensity analysis of OVA ingested by cells, analyzed by using Image $\mathrm{J}$ software. (E) A representative flow cytometry profile of nanoparticle uptake by DCs under different inhibitors, and $(F)$ representative fluorescence intensity histogram. ${ }^{*} p<0.05,{ }^{* *} p<0.01,{ }^{* *} p<0.001$.

A

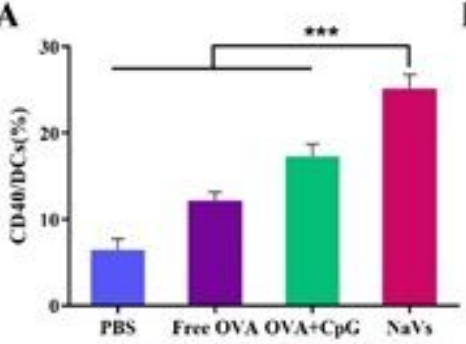

D

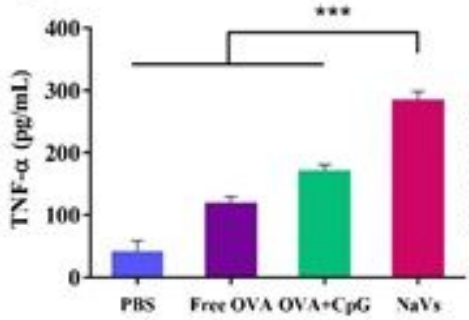

B

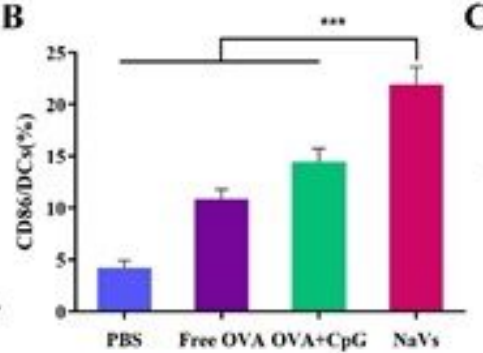

E
C

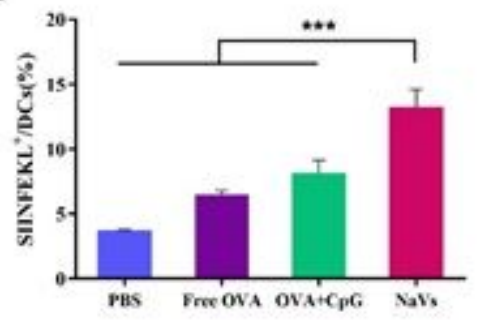

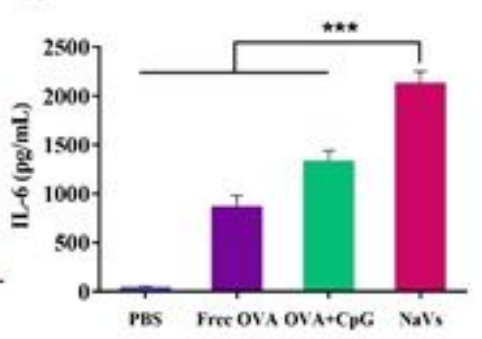

Figure 3 
Effects of different components on antigen cross-presentation and activation of BMDCs. Representative flow cytometry images of (A) CD40+ and (B) CD86+ and (C) SIINFEKL+ cells. Expression levels of (D) TNF- $a$ and (E) IL- 6 in supernatants were determined with ELISA assays, after BMDCs treated with PBS, Free OVA, OVA+CpG and NaVs. ${ }^{*} p<0.05,{ }^{* *} p<0.01,{ }^{* * *} p<0.001$.

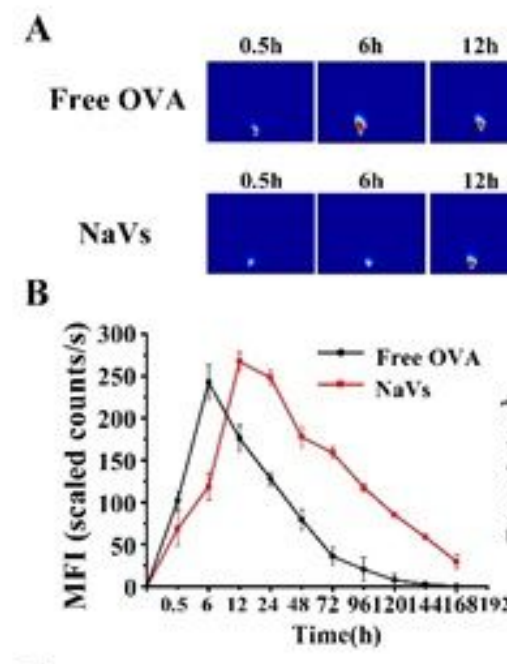

E

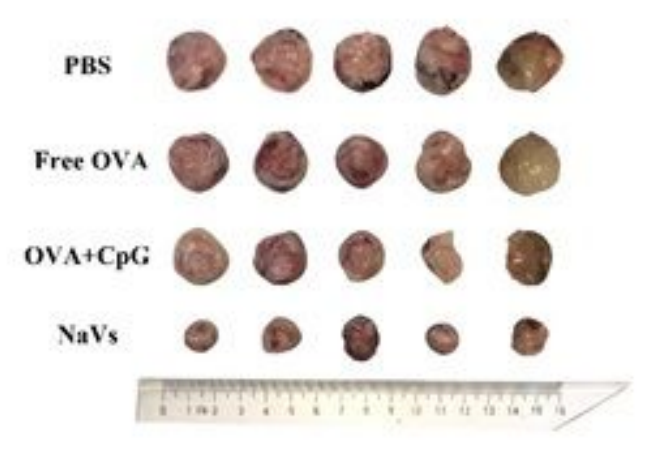

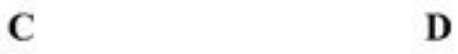

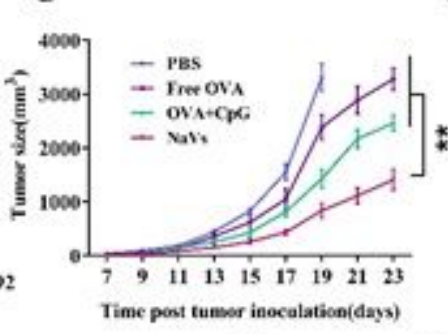

$\mathbf{F}$

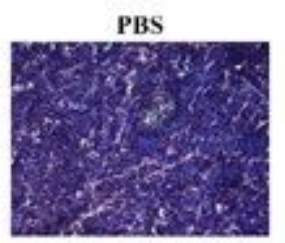

OVA+CpG

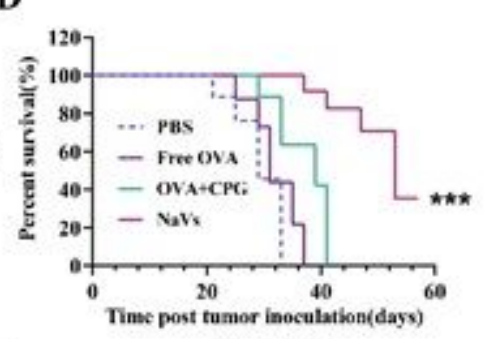

Free OVA

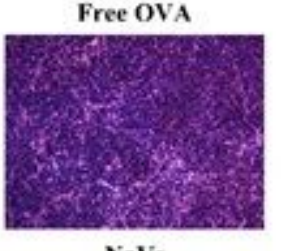

$\mathrm{NaVs}$

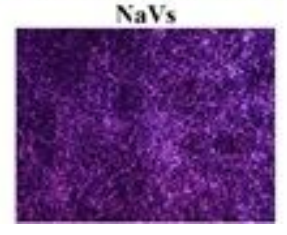

\section{Figure 4}

(A) Antigen release behavior of $\mathrm{NaVs}$ at the injection site. Representative fluorescence images of different formations at different points in time after injection. (B) Average fluorescence intensity at the injected site within a predetermined time period. (C) Average tumor growth curves were recorded and plotted. (D) Survival of mice treated with PBS, Free OVA, OVA +CpG or NaVs. (E) Tumors harvested from tumorbearing mice after different component treatments, $(F)$ and H\&E staining of tumor tissues obtained from mice immunized with different formulations. ${ }^{*} p<0.05,{ }^{\star \star} p<0.01$, ${ }^{\star \star \star} p<0.001$. 
A

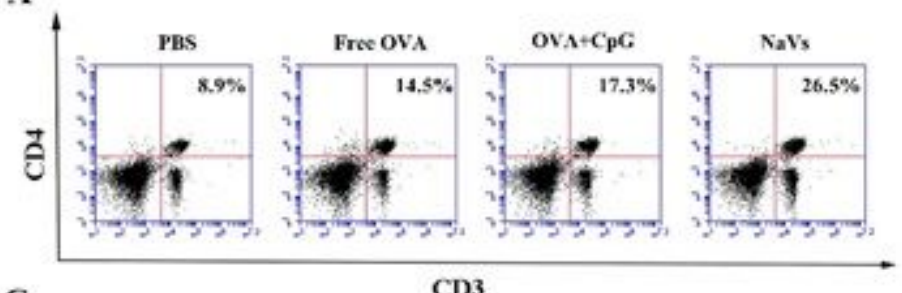

C

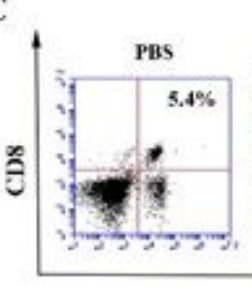

E

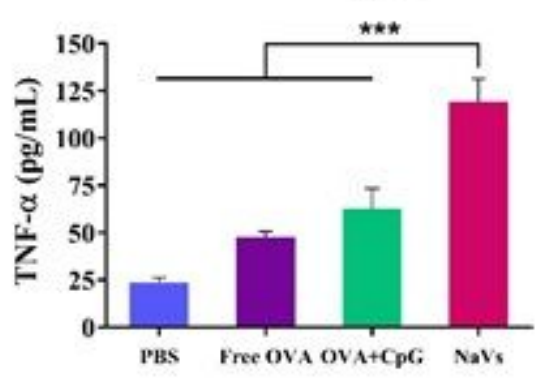

G

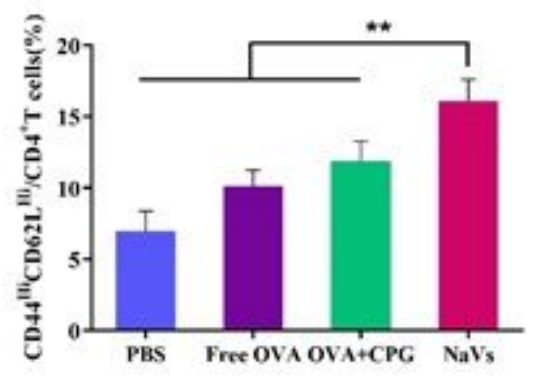

B
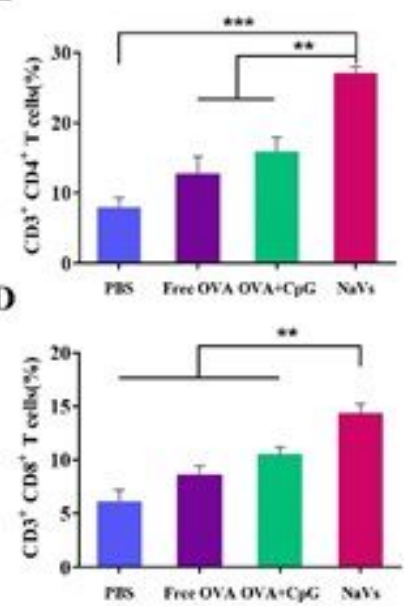

F

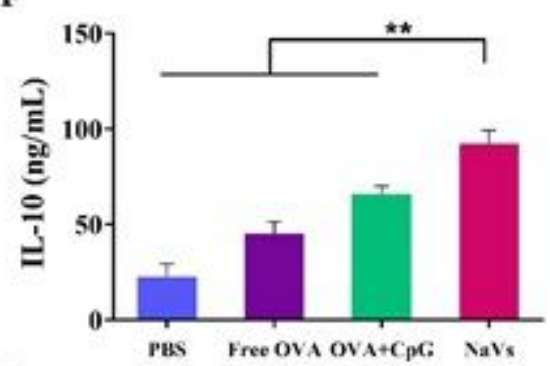

H

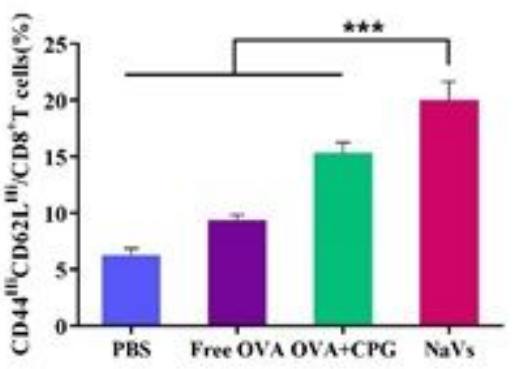

Figure 5

(A) Representative FACS plots and (B) percentage of CD3+CD4+ T cells and (C, D) CD3+CD8+ T cells were plotted for mice of different groups. And followed by histograms of (E) TNF-a and (F) IL-10. The type and proportion of memory $T$ cells in splenocytes after stimulation were detected and analyzed, and the proportion of memory $\mathrm{T}$ cells in $(\mathrm{G}) \mathrm{CD} 4+\mathrm{T}$ cell population or $(\mathrm{H}) \mathrm{CD} 8+\mathrm{T}$ cell population were shown. ${ }^{*} \mathrm{p}$ $<0.05,{ }^{*} \mathrm{p}<0.01,{ }^{* *} \mathrm{p}<0.001$ 


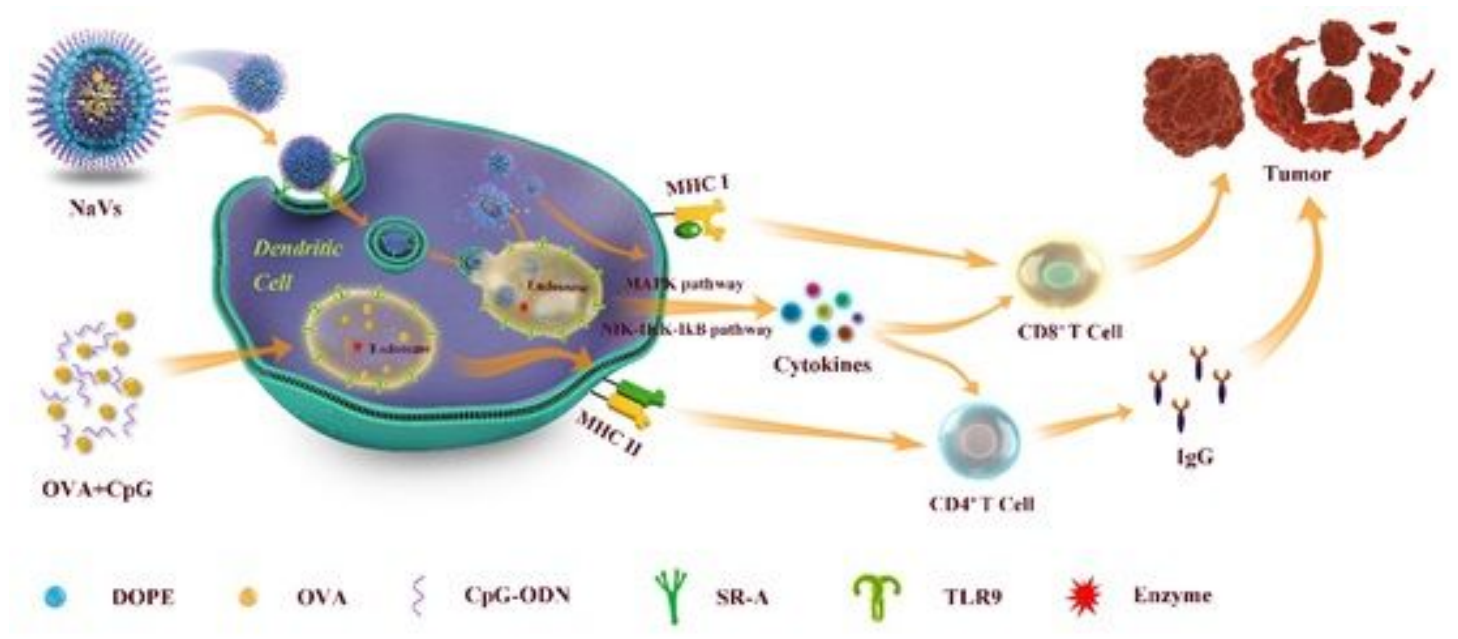

\section{Figure 6}

Illustration of NaVs anti-tumor immune effect mechanism.

\section{Supplementary Files}

This is a list of supplementary files associated with this preprint. Click to download.

- Supplementaryinformation.docx 